\title{
Vaccin contre le COVID-19: res- ponsabilités et droits des patients
}

\author{
Ursina Pally Hofmann \\ Dr iur., avocate, Secrétaire générale et cheffe du Service juridique de la FMH
}

\section{Bases légales de la responsabilité}

\section{Vue d'ensemble}

En matière de vaccination, le droit en vigueur considère qu'il existe principalement deux responsables primaires: le fabricant du vaccin et le médecin qui l'administre. Ce dernier contribue également à la mise en œuvre du plan de vaccination national.

Les considérations ci-après ont pour but d'expliquer comment sont réglées les questions de responsabilité civile, et de vous informer quant à vos obligations.

Dans le cas des vaccinations ordonnées ou recommandées par les autorités de santé, une responsabilité subsidiaire pour les dommages vaccinaux entre en ligne de compte, dès lors qu'il n'est pas possible de faire valoir des dommages-intérêts auprès du responsable primaire.

\section{Responsabilité du fait des produits}

La Loi sur la responsabilité du fait des produits [1] prévoit que le producteur répond du dommage lorsqu'un produit défectueux cause par ex. la mort d'une personne ou provoque chez elle des lésions corporelles. Par producteur, on entend toute personne - physique ou morale - qui est le fabricant d'un produit fini,

\section{Les attentes en matière de sécurité découlent des informations spécialisées et non des informations dans la notice accompagnant le médicament.}

le producteur d'une matière première ou le fabricant d'une partie composante. Il se peut également que des fournisseurs ou des personnes se présentant comme producteurs soient tenus pour responsables [2]. Par produit, on peut entendre notamment les médicaments [3]. Un produit est défectueux lorsqu'il n'offre pas la sécurité à laquelle on peut légitimement s'attendre compte tenu de toutes les circonstances. Les circonstances à prendre en compte sont la présentation du produit, l'usage pouvant en être raisonnablement attendu, et le moment de sa mise en circulation [4]. Les attentes en matière de sécurité pour les médicaments remis sur ordonnance - dont les vaccins - dé- coulent des informations spécialisées dont le médecin doit avoir connaissance et qu'il est tenu d'aborder avec le patient, et non des informations à l'intention du patient contenues dans la notice accompagnant le médicament [5]. La responsabilité du fait des produits n'entre pas en ligne de compte lorsque l'information à l'usage des patients est inexistante ou insuffisante, dès lors que les informations à l'intention du spécialiste sont complètes.

La responsabilité se reporte ainsi sur le médecin qui administre le vaccin, si ce dernier n'a pas suffisamment informé le patient sur la base des informations destinées au spécialiste.

\section{Responsabilité contractuelle - responsabilité de droit public}

En vertu du droit privé, le médecin répond en principe de tout manque de diligence dans l'administration d'un traitement ou dans l'information du patient. Toutefois, dans la mesure où les conditions de sa responsabilité sont remplies, les dommages sont couverts par son assurance responsabilité civile professionnelle [6]. Dans le cas d'une vaccination ordonnée par les autorités, le médecin agit en tant qu'organe de mise en œuvre. Dans la mesure où la législation cantonale en matière de responsabilité le prévoit, la responsabilité de l'Etat est engagée.

La situation n'est pas claire pour ce qui est des responsabilités dans le cas d'une vaccination recommandée, puisqu'il s'agit d'une vaccination facultative.

Les différences entre responsabilité privée ou étatique ne jouent toutefois aucun rôle quant aux devoirs du médecin administrant les vaccins. Dans les deux cas, il est tenu de traiter le patient avec diligence et de l'informer suffisamment. La différence réside dans la prise en charge des dommages-intérêts, soit par l'assurance responsabilité professionnelle du médecin, soit par la collectivité.

\section{Responsabilité en cas de vaccination obligatoire illicite}

S'il existe un risque significatif pour des personnes particulièrement exposées qui exercent une activité 
particulière, les cantons peuvent déclarer obligatoires certaines vaccinations [7]. L'importance du risque est évaluée sur la base de divers critères tels que la gravité potentielle de la maladie, l'efficacité attendue de l'introduction d'une telle obligation et la situation épidémiologique [8].

L'obligation de vaccination doit avoir une durée limitée et ne peut pas être exécutée par contrainte physique [9]. C'est pourquoi, même si une telle mesure devait être introduite, il faudra continuer d'informer chaque patient

Indépendemmant du type de responsabilité, le médecin est tenu de traiter le patient avec diligence et de l'informer suffisamment.

des effets potentiels et d'obtenir son consentement éclairé avant d'administrer le vaccin. Ainsi, même en cas d'obligation, personne ne sera vacciné contre son gré. Si ces principes ne sont pas respectés et si les autres conditions - existence d'un dommage et d'une relation de causalité entre l'obligation de vaccination et le dommage - sont remplies, la responsabilité de l'Etat sera engagée conformément aux lois cantonales en la matière.

\section{Responsabilité en cas de manque de diligence dans l'administration d'un vaccin}

Les médecins sont tenus de participer à la mise en œuvre du plan de vaccination national dans le cadre de leur activité. Ils doivent informer du plan national les personnes concernées par les recommandations de vaccination. Ils doivent également les informer des risques de contagion ou de maladie pour les personnes non vaccinées. Enfin, ils sont tenus de mettre à leur disposition le matériel d'information officiel de la Confédération et des cantons [10].

\section{Responsabilité subsidiaire de la Confédéra- tion selon la Loi sur les épidémies}

La Loi sur les épidémies prévoit qu'une indemnisation peut être accordée pour les dommages consécutifs à une vaccination si celle-ci a été ordonnée ou recommandée par les autorités. L'indemnisation n'est toutefois versée que si le dommage, en dépit d'efforts raisonnables, ne peut pas être couvert autrement [11].

Lorsqu'un patient subit un dommage parce qu'il a été informé de manière insuffisante, parce que le vaccin a été administré de manière inadéquate ou parce que le vaccin utilisé était défectueux, une indemnisation au sens de la Loi sur les épidémies peut entrer en considération, pour autant que les prétentions du patient ne soient pas couvertes par l'assurance du médecin ou du fabricant du vaccin, ou que ces prétentions aient déjà été rejetées.

\section{Devoirs du médecin administrant le vaccin}

\section{Diligence}

Il n'est possible de faire valoir une responsabilité pour les dommages consécutifs à une vaccination que s'il peut être prouvé que le médecin n'a pas agi avec la diligence nécessaire et que le dommage est la conséquence de ce manque de diligence.

Il y a violation du devoir de diligence si le médecin néglige des contre-indications à la vaccination, s'il utilise un vaccin non autorisé ou non adéquat, s'il ne respecte pas les normes d'hygiène ou le dosage prévu. Un stockage inadéquat du vaccin peut par ailleurs entraîner une responsabilité dès lors qu'il a causé une atteinte à la santé.

\section{Consentement éclairé}

Une large majorité des éventuelles prétentions en responsabilité pourraient découler du fait que le médecin n'a pas ou pas suffisamment informé le patient, car d'un point de vue technique, la vaccination est un acte médical relativement peu exigeant, et il est dès lors le plus souvent assez simple pour le médecin de respecter son devoir de diligence. Pour le vaccin contre le COVID-19 aussi, on portera une attention particulière à l'obtention du consentement éclairé du patient.

D'une manière générale, il faut fournir au patient toutes les informations nécessaires concernant les propriétés et les risques inhérents au vaccin pour qu'il puisse donner son consentement en connaissance de cause [12]

Les informations doivent être fournies au patient dans un entretien individuel. Les formulaires d'information ne peuvent pas le remplacer.

Pour informer le patient, le médecin doit se fonder sur les informations fournies dans la notice à l'intention du spécialiste [13]. Il est également tenu de lui faire part des éventuelles réserves exprimées par les autorités de santé concernant le degré de sécurité du produit utilisé.

Il doit par ailleurs informer le patient, le cas échéant, que la vaccination est facultative, et s'il existe un risque de contamination de tiers (selon le vaccin utilisé, cette possibilité existe) [14].

Enfin, le médecin est tenu d'évoquer avec chaque patient la question de la nécessité et de l'utilité du vaccin dans sa situation particulière. Il cherchera à établir et à discuter avec lui les risques qu'il encourt du fait d'une infection par l'agent pathogène contre lequel le vaccin doit le protéger. 
Il doit l'informer non seulement des risques les plus courants mais également de ceux ne présentant qu'une faible probabilité de réalisation, dans la mesure où ils sont connus et pourraient avoir des conséquences graves.

Lorsque tous les risques et effets secondaires potentiels du vaccin ne sont pas connus, il faut également en informer le patient. Ce point revêt une importance particulière pour les vaccins dont on ne connaît pas encore les effets à long terme.

\section{Lorsque les risques d'effets secondaires à long terme ne sont pas connus, il faut en informer le patient.}

Les informations doivent être fournies au patient dans le cadre d'un entretien individuel. Les formulaires d'information peuvent compléter utilement l'entretien individuel, mais pas le remplacer. Ils seront complétés en fonction du déroulement de l'entretien. L'information au patient sera consignée par écrit, de manière à pouvoir en justifier dans l'éventualité d'une procédure en responsabilité.

Secrétariat

du Service juridique

FMH

Nussbaumstrasse 29

CH-3000 Berne 15

Tél. 0313591111

Fax 0313591112

lex[at]fmh.ch

\section{Conclusion}

Qu'il s'agisse de la responsabilité de droit privé ou de droit public, on cherchera toujours à reconstituer quel a été le comportement du médecin traitant. Celui-ci est donc tenu de s'assurer que le traitement est effectué avec toute la diligence voulue. Cela implique notamment qu'il prenne soin d'informer suffisamment le patient et d'obtenir son consentement éclairé. Il ne peut s'en assurer que s'il se charge lui-même d'informer le patient ou s'il sait que l'information a été planifiée et donnée par l'éventuel organisme public responsable de la vaccination, de manière à ce qu'il ne puisse pas en être tenu responsable.

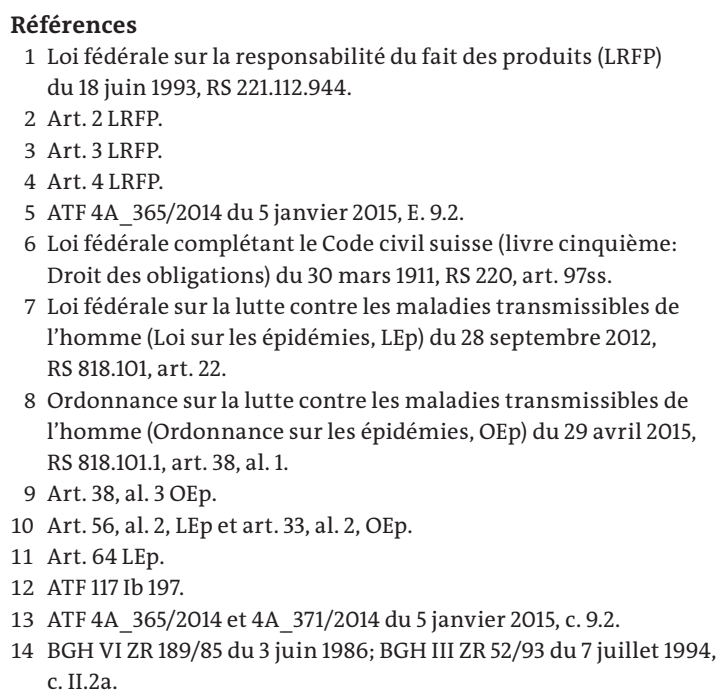

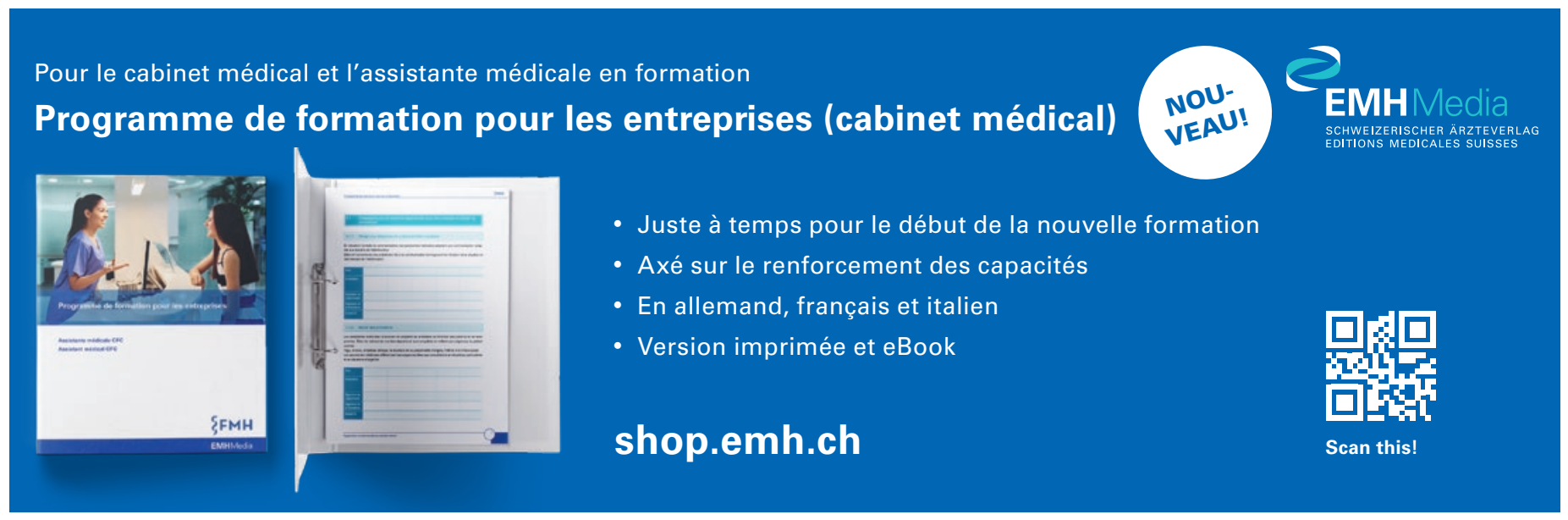

